\title{
A Dual Stopband Frequency Selective Surface for Mobile Shielding Applications
}

\author{
Aws Thamir Mayouf ${ }^{1}$, Khalil H. Sayidmarie ${ }^{2}$, and Yessar E. M. Ali ${ }^{3}$ \\ \{awsthamir@gmail.com ${ }^{1}$, kh.sayidmarie@gmail.com², a.yessar@yahoo.com ${ }^{3}$ \} \\ College of Engineering, University of Mosul, Mosul, Iraq ${ }^{1,3}$, \\ College of Electronic Engineering, Ninevah University, Mosul, Iraq ${ }^{2}$.
}

\begin{abstract}
A dual stopband frequency selective surface (FSS) for shielding two bands of the mobile frequencies is proposed. The designed FSS consists of periodic cells of concentric rings to prevent transmission of the two mobile frequencies of EGSM-900 and DCS-1800. The designed FSS is simulated in CST Microwave suit. The equivalent circuit for the two rings is analyzed, and an estimate for the frequency of the transmission in terms of the two stop frequencies is derived. The results show a stable response with various polarization angles. The obtained insertion loss is more than $30 \mathrm{~dB}$ across the two bands. This FSS can be used to isolate halls or reduce undesired radiation from nearby mobile base stations.
\end{abstract}

Keywords: frequency selective surface, shielding, dual band, equivalent circuit, multiresonance.

\section{Introduction}

The ever-increasing usage of mobile and wireless devices has led to many issues of spectrum managements such as shielding some areas from mobile frequencies or limiting other frequencies to a certain area. In many places and during various instances, the radiation from mobile phones can cause problems, where the signals cause interference, and the ringing tones become a source of annoyance. In examination halls and mosques, it is desirable to inactivate the mobiles inside the halls. This can be accomplished by either operating jamming devices, or shielding the hall from the mobile radiation. While the first can cause some health threats, and its use is illegal in many countries, the other measure may be achieved in principle by using the Faraday cage, which is costly and prevents other useful frequencies. The desired solution is a means that prevents unwanted frequency bands while permitting useful ones. This required filtering can be offered by the Frequency Selective Surfaces (FSS). Considering modern buildings that have large glass windows, the radio waves penetrate through the windows, which present lower attenuation than other external walls. Adding a properly designed FSS directly to the glass windows or as curtains will attenuate mobile phone waves and thus offers a simple solution.

The FSS are exhaustively studied in the literature [1] and implemented in many applications, such as shielding [2-8]. The various geometries that have been used in the unit cell of the FSS were rings of the square and circular shapes [2-6], crosses [7], hexagons [8], and U-shaped slots and strips [9]. 
When more than one band of frequencies are wanted to be treated, then the concept of multi-resonant elements in the shape of concentric rings [10] can be utilized in this application.

The shielding of the GSM frequencies by FSS in the form of wallpaper was reported [2], where the used unit cell of square loops with four inner rings provided attenuation of $20 \mathrm{~dB}$. A simulation study of a double-ring FSS showed more than 20dB shielding for the GSM frequencies [3]. A fractal square loop FSS with dual-band stop behavior etched on FR-4 substrate for the attenuation of GSM900, GSM1800, and IMT2000 frequency bands was presented in [4]. The unit cell geometry is a modification of the conventional square loop, which shows improved performance for oblique incidence up to $45^{\circ}$. A critical analysis of square, hexagonal, and circular FSS unit cells for a dual passband for GSM $900 \& 1800 \mathrm{MHz}$ frequencies was presented in [5]. A unit cell of the double circular ring was studied in [6], where the FSS was designed to attenuate the mobile bands of 0.8 , $0.9,1.8$, and $1.9 \mathrm{GHz}$, but transmits the Wi-Fi $2.45 \mathrm{GHz}$ band. The FSS was built on a float-glass to isolate the intensive care rooms of hospitals from mobile phone calls while leaving the Wi-Fi wave to pass through. An FSS for shielding the GSM 1800MHz downlink band was demonstrated in [7] to provide a $20 \mathrm{~dB}$ bandwidth of $133 \mathrm{MHz}$ and showed good angular and polarization performance. The FSS structure had circular slots to meet the requirement of ventilation and heat dissipation. A grid of hexagonal conducting rings printed on glass was also used to attenuate the propagating e.m. waves at $900 \mathrm{MHz}$ GSM and 2100MHz UTMS mobile frequencies [8]. The proposed FSS showed a stable response for incidence angles of up to $60^{\circ}$ while offering an attenuation of about $20 \mathrm{~dB}$ to transmitted waves.

This paper investigates an FSS unit cell of double square rings for the shielding of the GSM900 and GSM1800 bands. The design is intended for use as a curtain that will provide shielding of the e.m. wave at the required frequencies when it is dropped down. The analysis is based on the simulation using the CST microwave suit. The behavior of the spatial filtering by passing certain frequencies and blocking others is explained by examining the surface current density on the conducting rings. Moreover, an equivalent circuit for the two rings is proposed, and an estimate for the frequency of the transmission in terms of the two stop frequencies is derived.

\section{FSS Design}

The unit cell of the FSS was chosen as double concentric square rings that are known of their wideband or dual-band operation and similar response to both of the vertical and horizontal polarizations, as well as stability upon various incidence angles [10]. The standard frequency bands used in Iraq are the EGSM-900 (880-960 MHz) and the DCS1800 (1710-1880 MHz), and thus, the proposed FSS is to block these two bands.

Figure 1 shows the structure of the designed FSS element. The FSS structure is intended to be in the form of the fabric curtain, and the two rings are of copper strips printed of the fabric sheet. The curtain can be dropped down to work as e.m. shield, as it is an optical shield. The permittivity of woven fabrics is about 2.028 to 2.400 [11], and value of $\varepsilon_{r}=2.2$ was used in the simulations while the thickness of the curtain was assumed as $1 \mathrm{~mm}$. The resonance of the ring occurs when its average perimeter $\mathrm{L}_{\mathrm{av}}$ equals the guided wavelength $\lambda_{g}$, where 


$$
\mathrm{L}_{\mathrm{av}}=\lambda_{\mathrm{g}}=\frac{\lambda_{\mathrm{o}}}{\sqrt{\varepsilon_{\mathrm{eff}}}}
$$

Where $\varepsilon_{e f f}$ is the effective dielectric constant of the substrate (curtain) that is given by:

$$
\varepsilon_{\text {eff }}=\frac{\varepsilon_{\mathrm{r}}+1}{2}
$$

Table 1 shows the calculated dimensions of the double ring FSS, which is used to block the two GSM frequencies, where the center frequencies of $0.920 \mathrm{GHz}$ and $1.795 \mathrm{GHz}$ for the two GSM bands are used in the calculations. The outer and inner rings are designed to reject the lower and upper bands, respectively. The width of the double square rings was set to $1.4 \mathrm{~mm}$.

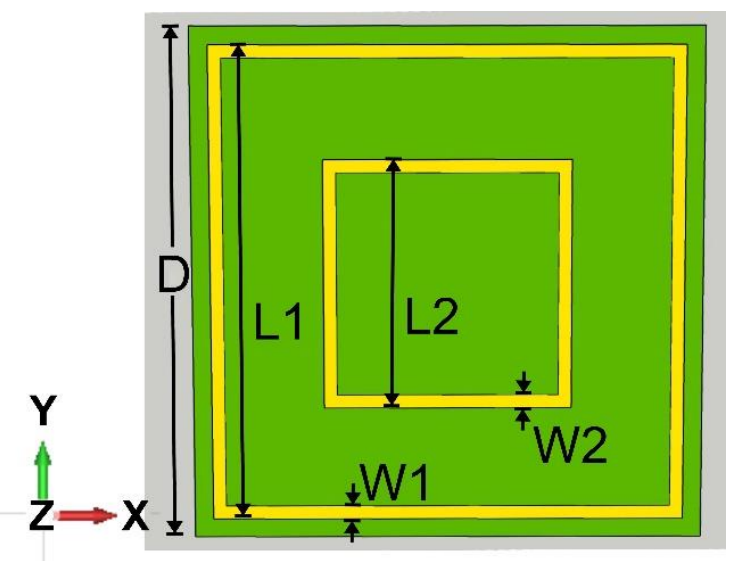

Fig. 1. The geometry of the FSS unit cell having double square rings.

\section{Simulation}

The CST Microwave Studio was used to investigate the performance and tune the dimensions of double rings that have been designed in the previous section. A linearly polarized wave is assumed normally-incident on the FSS, and its E-filed is along the Y-axis. The simulations started with a single square ring (the outer ring) whose S-parameters are shown in Figure 2. The response shows a band rejection at $0.942 \mathrm{GHz}$ frequency, $\left(S_{21}=-39.6 \mathrm{~dB}\right.$ and the $\left.S_{11} \approx 0 \mathrm{~dB}\right)$. As shown in Figure 3 and Figure 4, the surface current and E-field variations form one wavelength variation along the ring perimeter at the frequency of $0.9425 \mathrm{GHz}$. In terms of the wavelength calculation, the effective wavelength is $250.9 \mathrm{~mm}$, which is very near to the average perimeter of the ring is $254.4 \mathrm{~mm}$. Such variations indicate a resonating ring that blocks the transmission through the FSS. The dimensions of the ring that are obtained from the CST simulations to achieve rejection at the wanted band are listed in Table 1, and they very near to the calculated values. 
Table 1. The calculated dimensions compared to those found from the simulations.

\begin{tabular}{|l|l|l|l|l|l|}
\hline Parameter & $\mathrm{L}_{1}(\mathrm{~mm})$ & \multicolumn{1}{|c|}{$\mathrm{w}_{1}(\mathrm{~mm})$} & \multicolumn{1}{|c|}{$\mathrm{L}_{2}(\mathrm{~mm})$} & $\mathrm{w}_{2}(\mathrm{~mm})$ & $\mathrm{D}(\mathrm{mm})$ \\
\hline Calculated & 65.65 & 1.4 & 46 & 1.4 & 70 \\
\hline Found from CST & 65.5 & 1.4 & 46.5 & 1.4 & 70.5 \\
\hline
\end{tabular}

S-Parameters [Magnitude in dB]

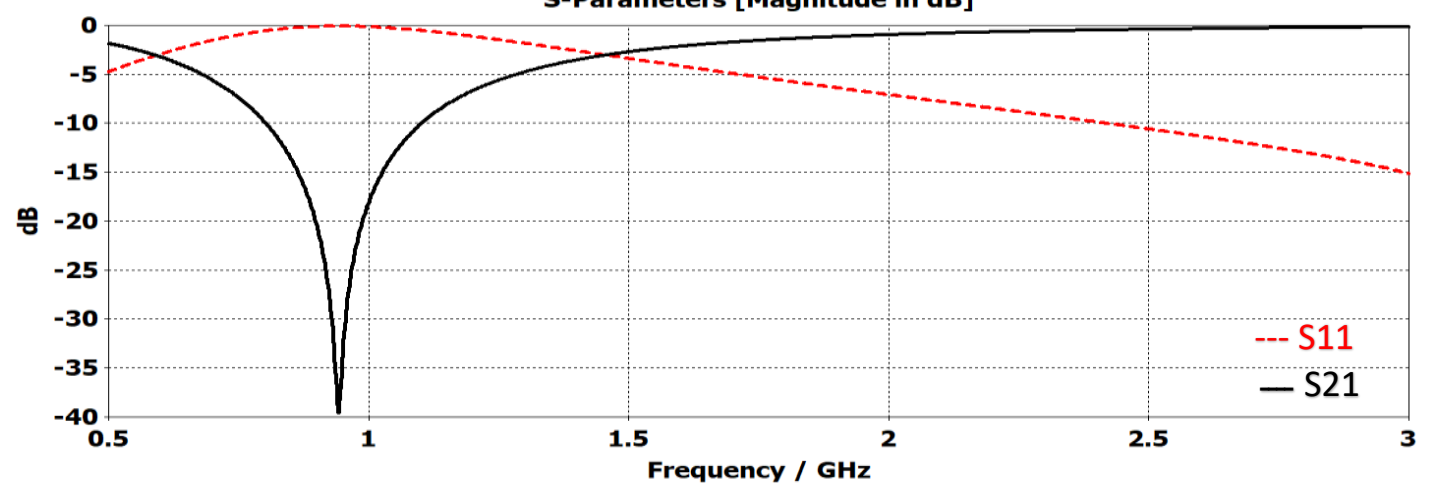

Fig. 2. The S-parameters of FSS having a single square ring of side length $65.5 \mathrm{~mm}$.

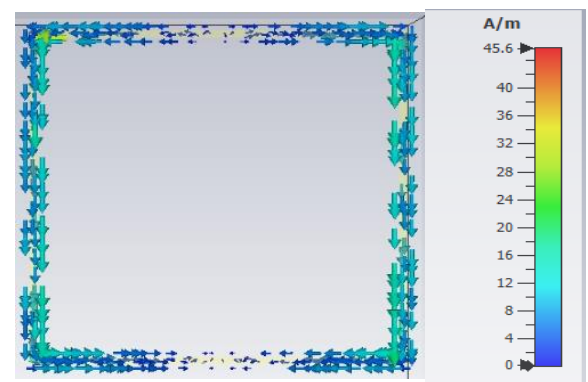

Fig. 3. The surface current distribution on the square ring at $0.9425 \mathrm{GHz}$ frequency
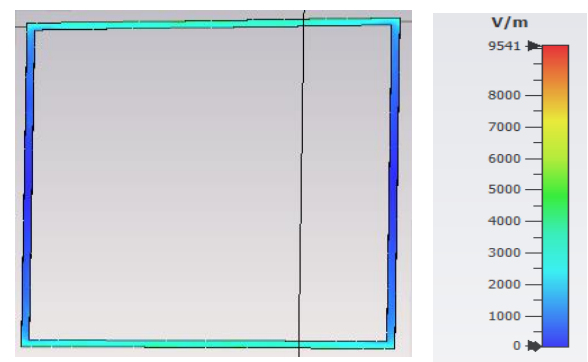

Fig. 4. The E-filed across the square ring at $0.9425 \mathrm{GHz}$ frequency 
The simulation results for the inner square ring alone are shown in Figure 5, which indicates the blocking of transmission at $1.79 \mathrm{GHz}\left(\mathrm{S}_{21}=-35.7 \mathrm{~dB}\right.$, and $\left.\mathrm{S}_{11} \approx 0 \mathrm{~dB}\right)$. The surface current variation along the ring (see Figure 6) shows about one wavelength change along the perimeter of the ring.

The combination of the two rings offers an FSS having blocked transmissions at the two desired bands, as shown in Figure 7. These two resonances correspond to the two rings. However, there is a small shift in the two former frequencies of $0.9425 \mathrm{GH}$ and $1.79 \mathrm{GHz}$ of the single ring cases to the new frequencies of $1.7825 \mathrm{GHz}$. This is attributed to the mutual coupling effect between the two rings.

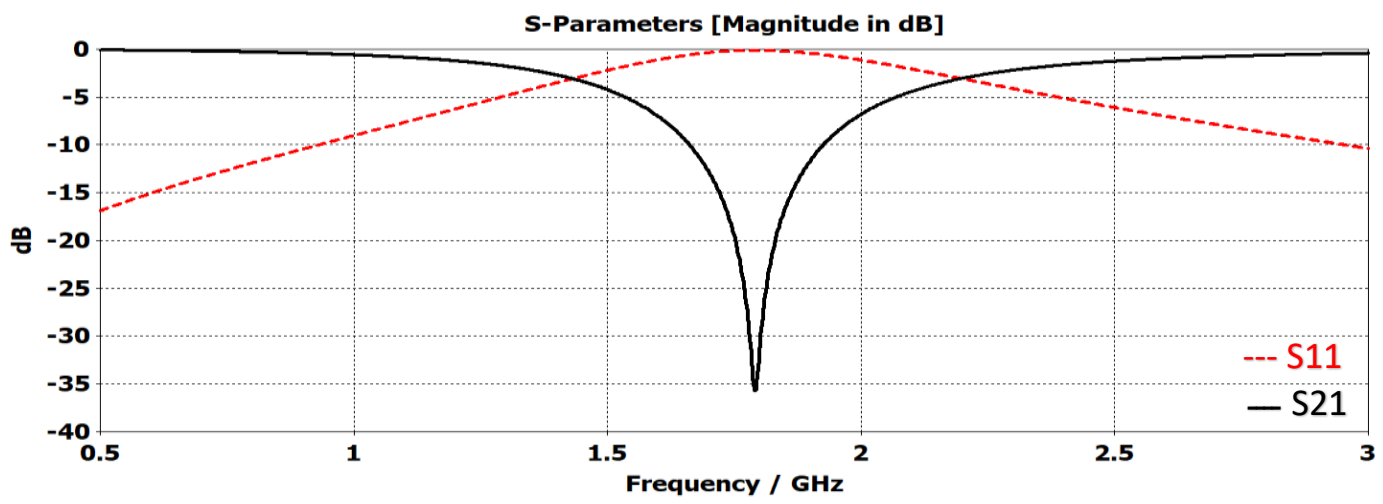

Fig. 3. S-parameters of the inner ring of $180.4 \mathrm{~mm}$ average perimeter
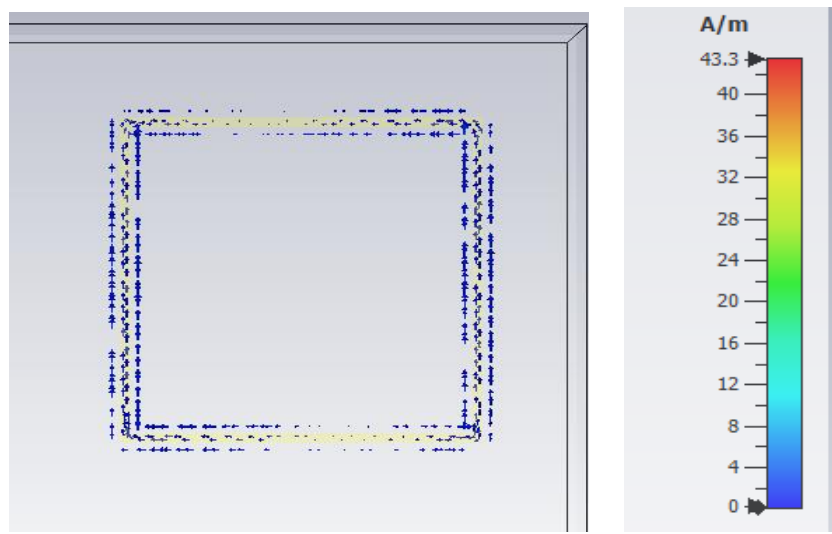

Fig. 4. The surface current distribution on the inner ring of $180.4 \mathrm{~mm}$ average perimeter at $1.79 \mathrm{GHz}$ frequency 
The current distribution on the rings at the frequency of $0.927 \mathrm{GHz}$ shows one wavelength change along the perimeter of the outer ring (Figure 8). The current density at the inner ring is smaller than that on the outer ring, indicating that the inner ring is not resonating at the above frequency. In addition, at the $1.782 \mathrm{GHz}$ frequency, one can note one wavelength change along the perimeter of the inner ring (Figure 9). The current density at the outer ring is smaller than that on the inner ring, indicating that the outer ring is not resonating at this frequency.

The other note about Figure 7 is that at a frequency of $1.327 \mathrm{GHz}$, there is no blocking of the incident wave, and the transmission coefficient is nearly unity $\left(S_{21} \approx 0 \mathrm{~dB}\right.$ and $\left.S_{11}=-30.7 \mathrm{~dB}\right)$. For further insight into this result, the current distribution on the dual rings at $1.327 \mathrm{GHz}$ is plotted in Figure 10. At this frequency, the currents on the two rings are in opposite directions, and this means that the effects of the currents on each side of the rings are almost eliminated. Thus, the transmission through the FSS at this frequency is explained. However, why transmission occurs at this frequency needs to be explained, as shown in the following.

The FSS, with its two resonating rings, can be modeled by an equivalent circuit comprising two parallel RLC circuits, as shown in Figure 11. Such an equivalent circuit was formerly used in [12] to explain the frequency response of FSS when the electromagnetics simulators were not available in 1983. The input impedance of the combined RLC resonators is given by:

$$
Z p=\frac{\left(1-\omega^{2} L_{1} C_{1}\right)\left(1-\omega^{2} L_{2} C_{2}\right)-\omega^{2} R_{1} R_{2} C_{1} C_{2}}{2-\omega^{2}\left(L_{1} C_{1}+L_{2} C_{2}\right)+j \omega\left(R_{1} C_{1}+R_{2} C_{2}\right)}
$$

And for low loss or lossless circuit $(\mathrm{R} 1=\mathrm{R} 2=0)$, then the impedance reduces to:

$$
\mathrm{Zp}=\frac{\left(1-\omega^{2} \mathrm{~L}_{1} \mathrm{C}_{1}\right)\left(1-\omega^{2} \mathrm{~L}_{2} \mathrm{C}_{2}\right)}{2-\omega^{2}\left(\mathrm{~L}_{1} \mathrm{C}_{1}+\mathrm{L}_{2} \mathrm{C}_{2}\right)}
$$

The two zeros of $Z_{p}$ which represent blocking of the transmission (high reflection) occur at the frequencies of:

$$
\begin{aligned}
& \omega_{1}=1 / \sqrt{ } \mathrm{L}_{1} \mathrm{C}_{1}, \\
& \omega_{2}=1 / \sqrt{ } \mathrm{L}_{2} \mathrm{C}_{2},
\end{aligned}
$$

While there is a single pole that occurs at the frequency $\omega_{\mathrm{t}}$ given by

$$
\omega_{\mathrm{t}}=\sqrt{2} \frac{\omega_{1} \omega_{2}}{\sqrt{\omega_{1}^{2}+\omega_{2}^{2}}}
$$

At the frequency of $\omega_{\mathrm{t}}$ the impedance tends to infinity, which explains the transmission through the FSS. Considering the results shown in Figure $7, \mathrm{f}_{1}=0.927 \mathrm{GHz}$, and $\mathrm{f}_{2}=1.782 \mathrm{GHz}$, then applying Eq. 7, the transmission frequency will be $\omega t=1.645 \mathrm{GHz}$. This represents a $12 \%$ error in the estimation of the transmission frequency. 


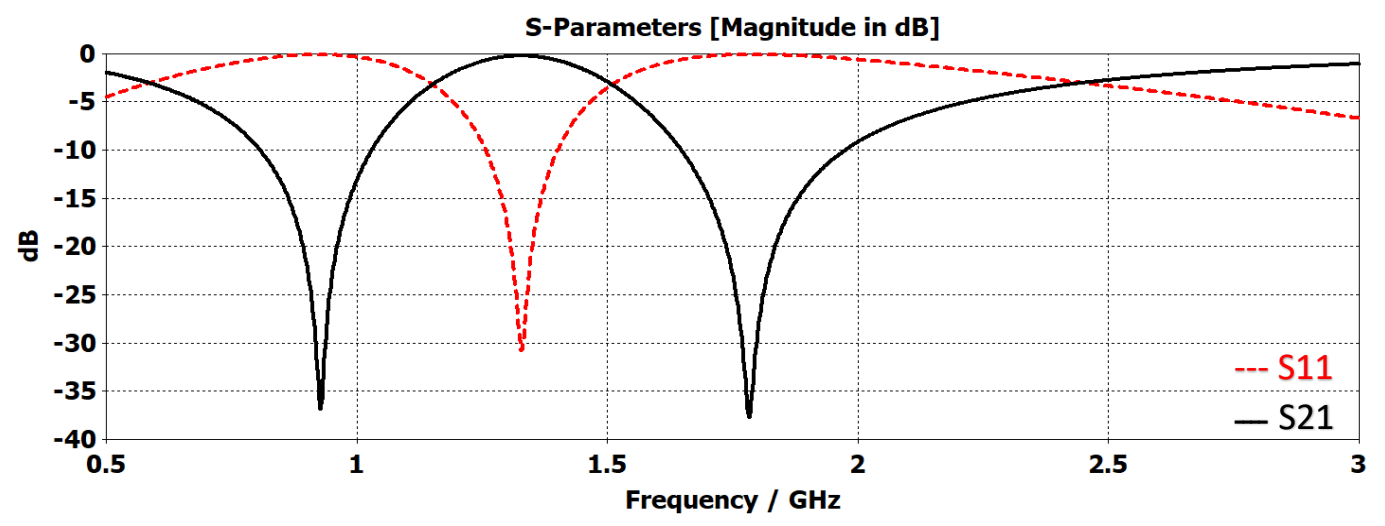

Fig. 7. The S-parameters of the double square ring when the E-filed is along the Y-axis.
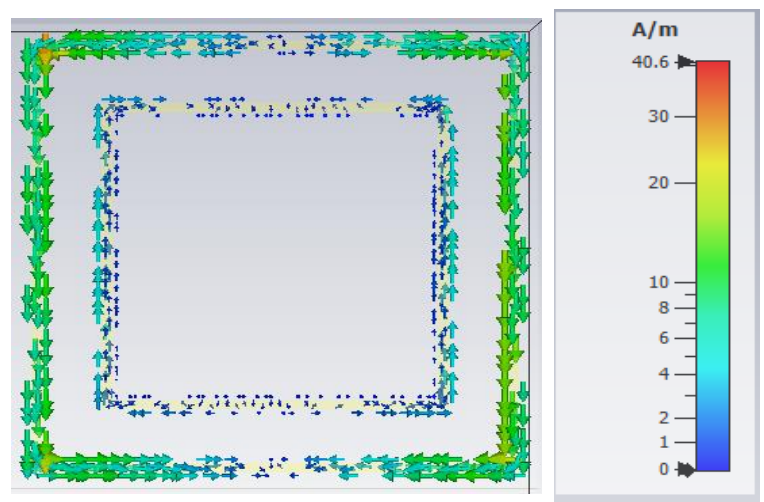

Fig. 5. The surface current on the double square ring at $0.927 \mathrm{GHz}$ frequency.
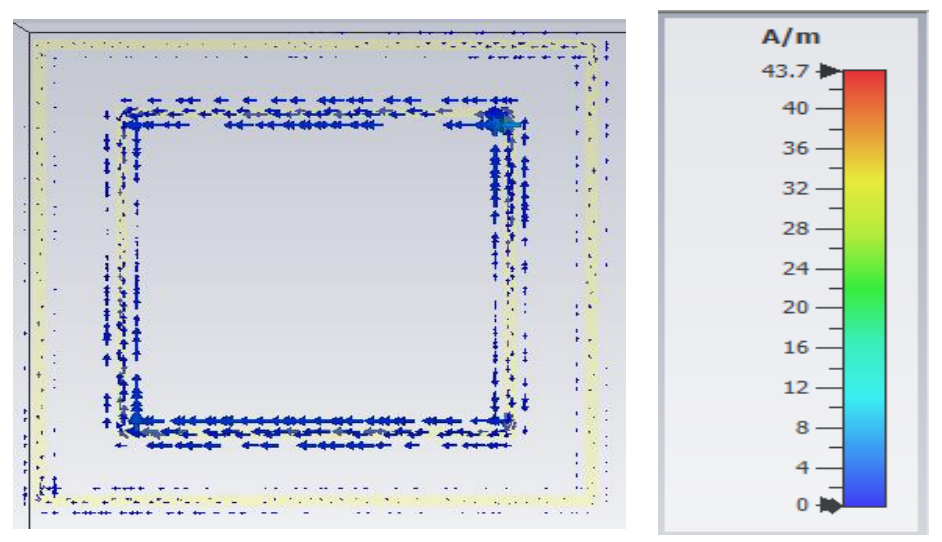

Fig. 6. The surface current on the double square ring at $1.782 \mathrm{GHz}$ frequency. 

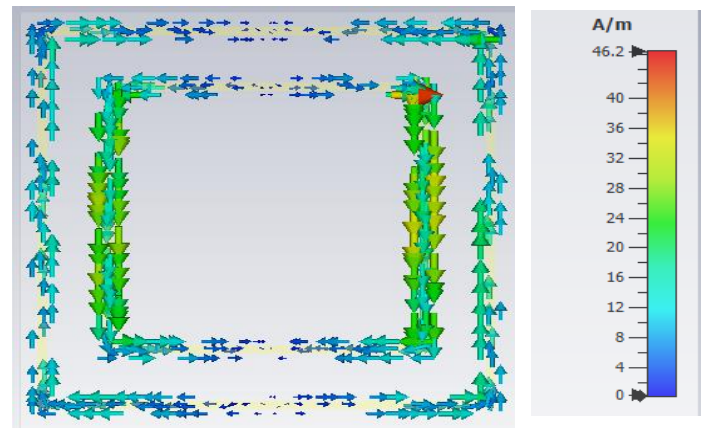

Fig. 7. The surface current on the double square ring at $1.327 \mathrm{GHz}$ frequency.

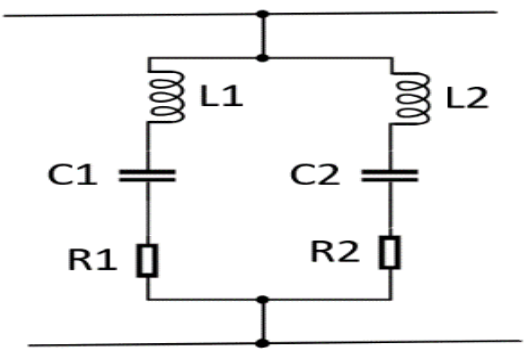

Fig. 11. The equivalent circuit for the double square rings.

For further study of the transmission case, two simulations were performed using two different perimeters for the inner ring while keeping the size of the outer ring fixed. Table 2 shows the calculated effective wavelength at each of the null frequencies. It can be seen that the blocking at the first band occurs when the perimeter of the outer ring equals the corresponding wavelength (1st and 3rd columns). The same Table (columns 2 and 4) also shows that the blocking at the upper band occurs when the effective wavelength is nearly equal to the perimeter of the inner ring.

While keeping the wave normally incident, the E-field was rotated by $45^{\circ}$ and $90^{\circ}$ to assess the FSS performance under various angles of the linear polarization, and the results are shown in Figure 12. The figure shows that the response of the FSS structure exhibit small changes, indicating that the square shape of the rings has little sensitivity to the angle of polarization. Thus, it can be concluded that this shape is also proper for circular polarization. The lower band is less affected by the change in the polarization angle as compared to the upper band.

Table 1. Performance of the double ring for various perimeters of the inner ring for an outer ring of fixed size.

\begin{tabular}{|l|l|l|l|l|l|}
\cline { 3 - 5 } \multicolumn{2}{l|}{} & \multicolumn{3}{c|}{ Effective wavelength at the ith null } \\
\hline$\#$ & Outer perimeter & Inner perimeter & $\begin{array}{l}1^{\text {st }} \text { band } \\
\text { Blocked })\end{array}$ & $2^{\text {nd }}$ (transmission) & $3^{\text {rd }}($ Blocked $)$ \\
\hline 1 & $256.4 \mathrm{~mm}$ & $180.4 \mathrm{~mm}$ & $255.5 \mathrm{~mm}$ & $178.5 \mathrm{~mm}$ & $132.9 \mathrm{~mm}$ \\
\hline 2 & $256.4 \mathrm{~mm}$ & $140.4 \mathrm{~mm}$ & $254.8 \mathrm{~mm}$ & $140.8 \mathrm{~mm}$ & $100.8 \mathrm{~mm}$ \\
\hline 3 & $256.4 \mathrm{~mm}$ & $116.4 \mathrm{~mm}$ & $257.6 \mathrm{~mm}$ & $117.9 \mathrm{~mm}$ & $83.1 \mathrm{~mm}$ \\
\hline
\end{tabular}




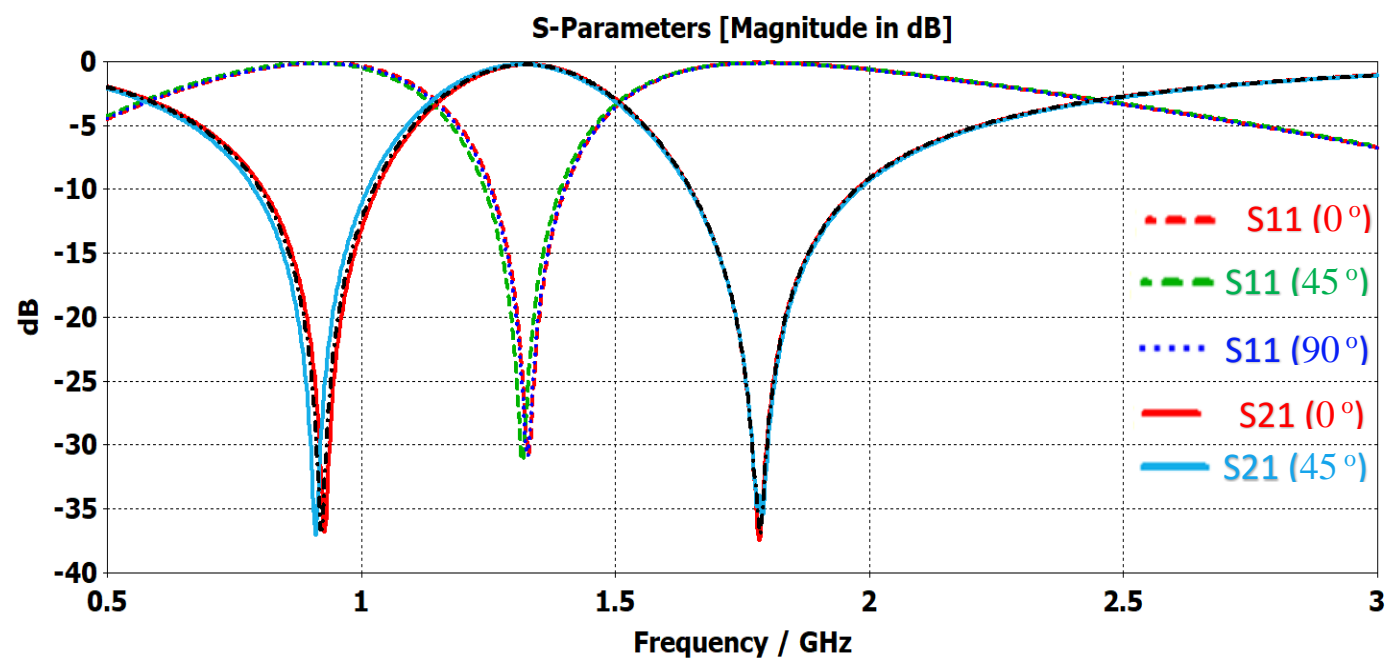

Fig. 12. The S-parameters of the double square ring when the linear polarization vector is at $0^{\circ}$, $45^{\circ}$ and $90^{\circ}$ with the Y-axis.

\section{Conclusion}

A single-layer FSS formed of two concentric square rings has been presented to offer shielding of the GSM900/1800 MHz frequency bands. The FSS is built by printing copper rings on a fabric sheet that represents a curtain for offering a shield across windows. The CST simulations show an attenuation of better than $30 \mathrm{~dB}$ at the two GSM bands. The FSS was represented by two parallel resonating circuits simulating the two rings. An estimate for the frequency of the transmission is proposed.

\section{References}

[1] B. Munk: Frequency Selective Surfaces: Theory and Design (2000).

[2] W. Kiermeier and E. Biebl: New Dual-Band Frequency Selective Surfaces for GSM Frequency Shielding. pp. 222-225.in Proceedings of the 37th European Microwave Conference, EUMC. (Oct. 2007)

[3] U. Rafique, S. A. Ali, M. T. Afzal, and M. Abdin: Bandstop Filter Design for GSM Shielding Using Frequency Selective Surfaces. vol. 2, no. 6, pp. 846-850. Int. J. Electr. Comput. Eng. (2012).

[4] F. C. Seman and N. K. Khalid: Investigations on Fractal Square Loop FSS at Oblique Incidence for GSM Applications. pp. 62-66. in Proceedings - 2014 Electrical Power, Electronics, Communications, Control, and Informatics Seminar, EECCIS 2014. In conjunction with the 1st Joint Conference UB-UTHM, (2014) 
[5] J. Singh, M. Najim, V. Agarwala, D. Singh, and G. D. Varma: Critical Analysis of Frequency Selective Surfaces for Dual Band GSM-900 \& 1800 MHz Transmission. pp. 207-210. in RAECE 2015 Conference Proceedings, National Conference on Recent Advances in Electronics and Computer Engineering. (2016)

[6] S. I. Sohail, "Wi-Fi Transmission and Multi-band Shielding Using Single-Layer Frequency Selective Surface,” 2016 IEEE Int. Symp. Antennas Propag., pp. 963-964, (2016).

[7] R. Sivasamy, M. Kanagasabai, S. Baisakhiya, R. Natarajan, J. K. Pakkathillam, and S. Palaniswamy: A Novel Shield for GSM 1800 MHz Band Using Frequency Selective Surface. vol. 38, pp. 193-199. Prog. Electromagn. Res. Lett. (2013).

[8] Y. Manaa, and R.W. Aldhaheri: Dual-Band frequency selective surface for GSM Shielding In Modern Buildings. Special Issue 14. Aljouf University Science and Engineering Journal.Scientific Conference in Surface Science and Its Applications CSSA (2017)

[9] M. A. Al-Atrakchii, K. H. Sayidmarie, R. A. Abd-Alhameed: Frequency Selective Surface Using the Metamaterial Property of the U-Shaped Strip. Accepted in the 2020-IMDC-SDSP Conference. Antalya, Turkey (8-10 April 2020)

[10] M.E. Bialkowski, and K. H. Sayidmarie: Investigations into phase characteristics of a single-layer reflectarray employing patch or ring elements of variable size. Vol. 56, No. 11, PP. 3366-3372. IEEE Transac. on Antennas and Propagation (2008)

[11] K. Bal and V. K. Kothari: Permittivity of woven fabrics: A comparison of dielectric formulas for air-fiber mixture.vol. 17, no. 3, pp. 881-889. IEEE Trans. Dielectr. Electr. Insul. (2010)

[12] R. J. Langley, and E. A. Parker: Double-Square Frequency-Selective Surfaces and Their Equivalent Circuit. Vol. 19, No. 1. Electronics Letters. (18 Aug. 1983). 